\title{
Studying thermal conductivity of magnetic nanofluids in constant magnetic field
}

\author{
Maxim Pryazhnikov ${ }^{1,2, *}$, Andrey Minakov ${ }^{1,2}$, Dmitriy Guzei $^{1,2}$, and Vladimir Zhigarev ${ }^{1}$ \\ ${ }^{1}$ Siberian Federal University, 660041, Krasnoyarsk, Russia \\ ${ }^{2}$ Institute of Thermophysics SB RAS, 630090, Novosibirsk, Russia
}

\begin{abstract}
The thermal conductivity of magnetic nanofluids in a constant magnetic field is investigated. The concentration dependence of the thermal conductivity coefficient of magnetic fluids based on water and iron oxide nanoparticles $\mathrm{Fe}_{3} \mathrm{O}_{4}$ in a constant magnetic field is obtained. The dependence of the thermal conductivity coefficient of nanofluid on the nanoparticles size in a magnetic field is obtained. The temperature effect on the thermal conductivity of magnetic nanofluid in a constant magnetic field is studied.
\end{abstract}

\section{Introduction}

Recently, ferromagnetic nanofluids are actively investigated (carrier fluid + iron oxides nanoparticles). A distinctive feature of ferromagnetic nanofluids is responsiveness to an external magnetic field. In the works of other researchers, the effect of increasing the thermal conductivity was observed. For example, in [1] was a $23 \%$ increase in the thermal conductivity of nanofluid based on kerosene and $\mathrm{Fe}_{3} \mathrm{O}_{4}$ nanoparticles $(10 \mathrm{~nm}$ in size and a $7.8 \%$ volume concentration), and $300 \%$ in the absence of a magnetic field. The authors of [2] investigated water-based nanofluids with $\mathrm{Fe}_{3} \mathrm{O}_{4}$ nanoparticles $(10 \mathrm{~nm}$ in size and $5 \%$ volume concentration) under the magnetic field action and observed a more than triple increase in thermal conductivity.

\section{Experimental apparatus and procedure}

Within the framework of our study we measured the ferromagnetic thermal conductivity of nanofluid in a constant magnetic field. $\mathrm{Fe}_{3} \mathrm{O}_{4}$ nanoparticles were used (nanoparticles powders of the «Plasmotherm», Moscow). Distilled water was base fluid. A standard twostep method was used to prepare nanofluids.

Measurement of thermal conductivity of nanofluids was carried out by the hot-wire method with a Wheatstone bridge circuit. Detailed description of the test bench and testing technique is given in [3]. Wheatstone bridge was used as the basis of the test bench instrumentation to measure an unknown electrical resistance of hot wire. Principle of measuring the thermal conductivity by the hot-wire method is based on a linear dependence

* Corresponding author: $\underline{\operatorname{arrivent} @ \text { yandex.ru }}$ 
between the temperature rise of hot wire and the logarithm of heating time of the wire. A copper wire with a length of $200 \mathrm{~mm}$ and a diameter of 75 microns was used in the experiments. The wire was immersed in a vessel of diameter $32 \mathrm{~mm}$ containing $200 \mathrm{ml}$ of the fluid under study.

Two permanent neodymium magnets with dimensions $50 \times 30 \times 10 \mathrm{~mm}$ were used to create a magnetic field. The magnetic field intensity around the magnet was measured with a milliteslameter TPU-02. The distribution of the magnetic field induction over the magnet is shown in figure 1. The maximum value of the magnetic induction near the magnet is of the order of $0.3 \mathrm{~T}$. In experiments, the magnets were located in the immediate vicinity of the measuring cell. Two cases of a magnetic field were considered (see figure 2): a magnetic field created by a single magnet (low magnetic field intensity) and a magnetic field created by two magnets (high magnetic field intensity).

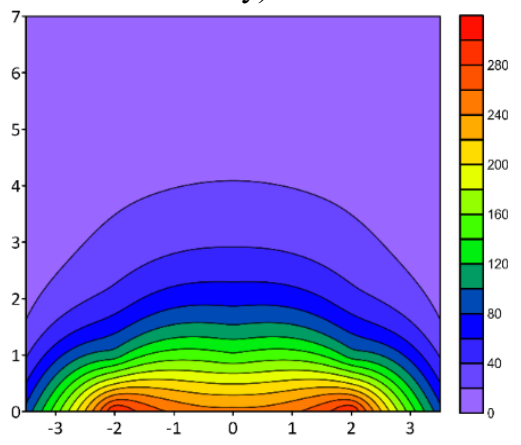

Fig. 1. The magnetic field induction over the magnet $(\mathrm{mT})$.

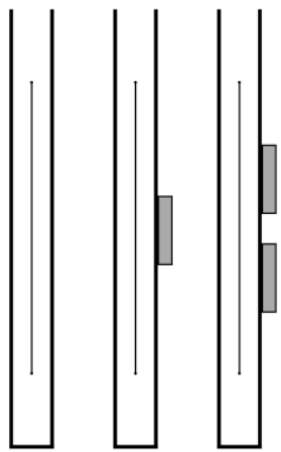

Fig. 2. The arrangement of magnets near the vessel.

\section{Results and discussion}

The effect of volume nanoparticles density on the thermal conductivity of nanofluid was studied. $\mathrm{Fe}_{3} \mathrm{O}_{4}$ nanoparticles of $50 \mathrm{~nm}$ average size were used. Two volume nanoparticles density in water of 1 and $2 \%$ are considered. It is found that the influence of magnetic field intensifies with increase of nanoparticles concentration. As a result of measurements the thermal conductivity of magnetic nanofluid is increased under the influence of magnetic field. For magnetic nanoparticles 2.2 and 2.8 times enhancement in thermal conductivity is achieved for $2 \%$ nanoparticle concentration by the application low and high magnetic field intensity, respectively (see figure $3 a$ ). The thermal conductivity of magnetic nanofluid intensifies with increase of magnetic field intensity (see figure $3 a$ ). It should be noted that 
with increasing magnetic field intensity, an increase and decrease in the thermal conductivity of a magnetic nanofluids can be observed $[5,6]$.

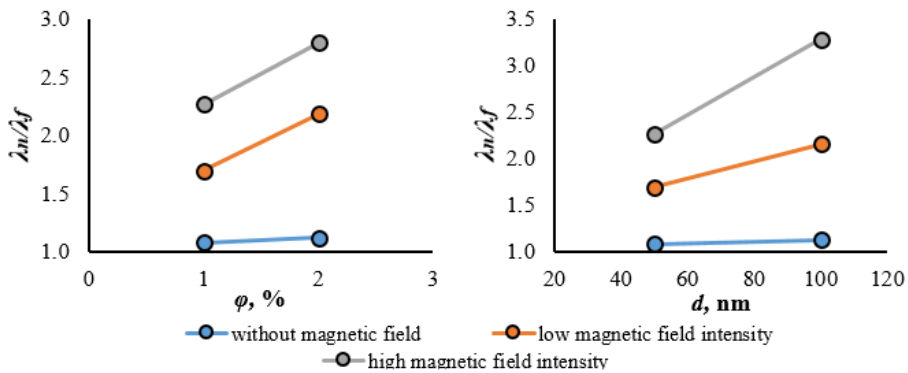

Fig. 3. Dependence of relative thermal conductivity coefficient of nanofluids on nanoparticle concentration $(a)$ and on nanoparticle size $(b)$.

It is established that the influence of magnetic field intensifies with increase of nanoparticles concentration. The thermal conductivity coefficient intensifies by $5 \%$ with an increase the nanoparticles concentration from 1 to $2 \%$ without a magnetic field, while increase amounts to $30 \%$ under the influence of the magnetic field (see figure $3 a$ ).

The nanoparticle size effect on the thermal conductivity of nanofluid in a magnetic field was investigated. The study was carried out for $\mathrm{Fe}_{3} \mathrm{O}_{4}$ nanoparticles. The volume nanoparticles density in water was $1 \%$. Nanoparticles powders of 50 and $100 \mathrm{~nm}$ average size were considered. It is found that the thermal conductivity of nanofluid increases with the nanoparticles size, both under the influence of the magnetic field and without it. It was shown for the first time that the thermal conductivity coefficient of nanofluid increases under the influence of a magnetic field with increasing particle size.

The influence of nanoparticle size on the thermal conductivity of nanofluid in magnetic field is studied. The volume nanoparticles density in distilled water was $1 \%$. Figure $3 \mathrm{~b}$ shows the dependence of the relative thermal conductivity coefficient of the nanofluid (the ratio of the thermal conductivity coefficient of nanofluid to the thermal conductivity coefficient of base fluid) on the nanoparticles size in water.

It is obtained that the thermal conductivity coefficient of nanofluid increases with the nanoparticles size, both under the influence of the magnetic field and without it. Similar behavior was noted earlier for other nanofluids [4]. In absence of magnetic field the addition of nanoparticles with $50 \mathrm{~nm}$ average size to water increases the thermal conductivity by $8 \%$ in comparison with the thermal conductivity coefficient of water, and nanoparticles of $100 \mathrm{~nm}$ size increase by $13 \%$. Increment becomes larger with the application of magnetic field. The thermal conductivity of nanofluid with nanoparticles of size $50 \mathrm{~nm}$ at low magnetic field intensity is $70 \%$ higher than the thermal conductivity of water, and $11 \%$ with $100 \mathrm{~nm}$ nanoparticles. In a magnetic field intensity created by two magnets, the growth is 2.27 times for fine nanoparticles $(50 \mathrm{~nm})$ and 3.28 times for large nanoparticles $(100 \mathrm{~nm})$. Thus, it was shown for the first time that the thermal conductivity coefficient of nanofluid intensifies with an increase particle size under the influence of the magnetic field.

The temperature dependence of the thermal conductivity of nanofluid in a magnetic field is studied. The investigation was carried out for $\mathrm{Fe}_{3} \mathrm{O}_{4}$ nanoparticles $(50 \mathrm{~nm})$. The range of the investigated temperatures is $25-50^{\circ} \mathrm{C}$. The dependence of the relative thermal conductivity coefficient of nanofluid on temperature is obtained (see figure 4): the relative thermal conductivity coefficient of a nanofluid without a magnetic field is 1.08 at a temperature of $25^{\circ} \mathrm{C}$, it increases linearly with temperature rise and reaches a value of 1.15 at $50^{\circ} \mathrm{C}$. 


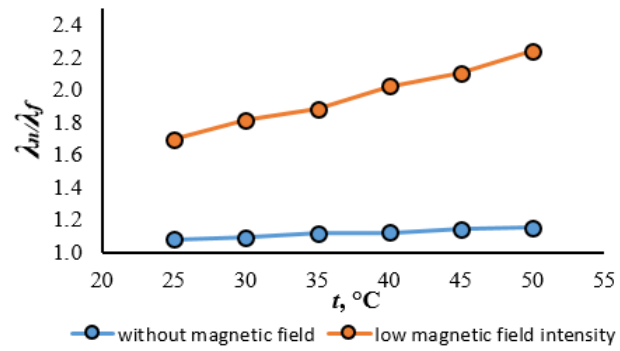

Fig. 4. Dependence of the relative thermal conductivity coefficient of nanofluid on temperature.

The magnetic field effect on temperature dependence of thermal conductivity of magnetic nanofluid is studied. It is shown that the magnetic field effect on thermal conductivity of magnetic nanofluid increases with temperature rise of nanofluid. It should be noted that in $[2,6]$ it was shown that with increasing temperature, the coefficient of thermal conductivity decreases.

\section{Conclusions}

The thermal conductivity of magnetic nanofluids in a constant magnetic field is investigated. The concentration dependence of the thermal conductivity coefficient of magnetic fluids based on water and iron oxide nanoparticles $\mathrm{Fe}_{3} \mathrm{O}_{4}$ in a constant magnetic field is obtained. The dependence of thermal conductivity coefficient of nanofluids on the nanoparticles size in a magnetic field is obtained. As a result of measurements the thermal conductivity of magnetic nanofluid is increased under the influence of magnetic field. The thermal conductivity of magnetic nanofluid intensifies with increase of magnetic field intensity (see figure 3).

The magnetic field effect on temperature dependence of thermal conductivity of magnetic nanofluid is studied. It is shown that the magnetic field effect on thermal conductivity of magnetic nanofluid increases with temperature rise of nanofluid (see figure 4).

The current work is performed at partial support of the projects funded by the Russian Foundation for Basic Research and Krasnoyarsk Regional Fund for Support of Scientific and Scientific-Technical Activities (Contract No. 16-48-243061).

\section{References}

1. J. Philip, P.D. Shima, B. Raj, Appl. Phys. Lett. 91, 203108 (2007).

2. A. Gavili, F. Zabihi, T.D. Isfahani, Sabbaghzadeh, J. Exp. Therm. Fluid Sci. 41, 94 (2012).

3. A.V. Minakov, M.I. Pryazhnikov, V.Ya. Rudyak, D.V. Guzei, A.S. Lobasov, J. Eng. Phys. Thermophys. 88, 149 (2015).

4. M. Pryazhnikov, A. Minakov, V. Rudyak, D. Guzei, Int. J. Heat Mass Transfer. 104, 1275 (2017).

5. C.L. Altan, A. Elkatmis, M. Yüksel, N. Aslan, S. Bucak, J. App. Phys. 110, 093917 (2011).

6. A. Karimi, M. Goharkhah, M. Ashjaee, M.B. Shafii, Int. J. Thermophys. 362720 (2015). 